\title{
Discourse Representation Theory 1090
}

\author{
Jan van Eijck
}

\author{
CWI, Kruislaan 413, 1098 SJ Amsterdam, The Netherlands \\ OTS, Trans 10, 3512 JK Utrecht, The Ne therlands
}

February 15, 2005

\begin{abstract}
Discourse Representation Theory is a specific name for the work of Hans Kamp in the area of dynamic interpretation of natural language. Also, it has gradually become a generic term for proposals for dynamic interpretation of natural language in the same spirit. These proposals have in common that each new sentence is interpreted in terms of the contribution it makes to an existing piece of interpreted discourse. The interpretation conditions for sentences are given as instructions for updating the representation of the discourse.

This article first introduces the problem that discourse representation theory, in its specific sense, sets out to solve. Then the basic ideas of the theory are listed, various extensions of the basic theory are discussed, the relation to partial interpretation of language is sketched, and proof theory for discourse representation structures is presented. The paper ends with a brief account of the use of 'unresolved' discourse representation structures for the representation of ambiguities.
\end{abstract}

\section{The Problem of Unbound Anaphora}

The most straightforward way to establish links between anaphoric pronouns and their antecedents is to translate the pronouns as variables bound by their antecedents. This approach does not work when the link crosses a sentence boundary, as in example (1).

A man $_{1}$ met an attractive woman $_{2}$. He smiled at her $e_{2}$.

It should be possible to interpret the first sentence of this discourse as soon as it is uttered, and then later on, while processing the second sentence, establish the links between the pronouns and their intended antecedents. 
One possible solution is translating the indefinites by means of existential quantifiers with scopes extending beyond the sentence level, and then allow the variables for the pronouns to be captured by these quantifiers. But this will not do: at some point the scope of a quantifier has to be 'closed off', but further on another pronoun may occur that has to be linked to the same antecedent.

The bound variable approach to anaphora also fails for cases where a pronoun in the consequent of a conditional sentence is linked to an indefinite noun phrase in the antedent of the conditional, as in example (2).

$$
\text { If a } \text { man }_{1} \text { meets an attractive } \text { woman }_{2} \text {, he } e_{1} \text { smiles at her } 2 .
$$

A possible approach here would be to view (2) as a combination of the noun phrases a man and an attractive woman with a structure containing the appropriate gaps for antecedents and pronouns, viz. (3). This is the approach of quantifying-in, taken in traditional Montague grammar (see the article MONTAGUE GRAMMAR).

$$
\text { If } \mathrm{PRO}_{1} \text { man meets } \mathrm{PRO}_{2}, \mathrm{PRO}_{1} \text { smiles at } \mathrm{PRO}_{2} \text {. }
$$

This approach does not work here, however. Quantifying-in the indefinite noun phrases in (3), i.e. in a structure that has the conditional already in place, would assign the wrong scope to the indefinites with respect to the conditional operator.

Note that the meaning of (2) is approximately the same as that of (4).

Every man who meets an attractive woman $_{1}$ smiles at her s. $_{1}$

In this case as well, quantifying-in does not allow one to generate the most likely reading where the subject of the sentence has wide scope over the enbedded indefinite. Sentences with the patterns of (2) and (4) have reached the modern semantic literature through Geach (1962). Geach's discussion revolves around examples with donkeys, so these sentences became known in the literature as donkey sentences.

As has repeatedly been remarked in the literature, there are quite striking structural parallels between nominal and temporal anaphora. The past tense can be viewed as an anaphoric element in all those cases where it is not to be understood as 'sometime in the past' but as referring to some definite past time.

John saw Mary. She crossed the steet.

In example (5), presumably the seeing takes place at some specific time in the past, and the crossing immediately after the seeing. Again, we have an anaphoric link across sentence boundaries, and a traditional operator approach to tense does not seem to fit the case. Although tense is not treated in the pioneer papers on discourse representation, it is clear that the problem of temporal anaphora is a very important subproblem of the general anaphora problem that discourse representation theory sets about to solve. 


\section{Basic Ideas}

Discourse representation theory as it was presented in Kamp (1981) addressed itself specifically at the problem of the previous section, although confined to nominal anaphora. The basic idea of the approach is that a natural language discourse (a sequence of sentences uttered by the same speaker) is interpreted in the context of a representation structure. The result of the processing of a piece of discourse in the context of representation structure $R$ is a new representation structure $R^{\prime}$; the new structure $R^{\prime}$ can be viewed as an updated version of $R$.

The interpretation of indefinite noun phrases involves the introduction of discourse referents or reference markers for the entities that a piece of discourse is about. In the following, the term discourse referent will be used. Discourse referents are essentially free variables. Thus, indefinite noun phrases are represented without using existential quantifiers. The quantification is taken care of by the larger context. It depends on this larger context whether an indefinite noun phrase gets an existential reading or not.

The life span of a discourse referent depends on the way in which it was introduced. All 'alive' referents may serve as antecedents for anaphors is subsequent discourse. Anaphoric pronouns are represented as free variables linked to appropriate antecedent variables. Definite descriptions in their simplest use are treated in a way which is similar to the treatment of anaphoric pronouns: definite noun phrases in their anaphoric use are treated like indefinite noun phrases, i.e. they are translated as free variables, but give rise to additional anaphoric links. The treatment of other, functional uses of definite noun phrases (as in $A$ car crashed. The driver emerged unhurt.) is more involved.

The difference between indefinite noun phrases on one hand and definite noun phrases and pronouns on the other, is that indefinites introduce new variables, whereas the variables introduced by definites and pronouns always are linked to an already established context. In other words, the difference between definites (including pronouns) and indefinites is that the former refer to entities that have been introduced before, i.e. to familiar entities, while the latter do not.

Quantifier determiners, i.e. determiners of noun phrases which are neither definite nor indefinite, can bind more than one variable. Specifically, they can bind a block of free variables some of which may have been introduced by indefinites. Conditional operators (if . . then ... constructions) can also bind blocks of free variables. Not all variables introduced by indefinites are in the scope of a quantifier or a conditional operator. Those which are not are existentially quantified over by default.

The processing of a piece of discourse is incremental. Each next sentence to be processed is dealt with in the context of a structure which results from processing the previous sentences. The processing rules decompose a sentence, replacing the various parts by conditions to be added to the structure. Assume one is processing discourse (6) in the context of representation structure (7) 
containing just one discourse referent and one condition.

A man walked down the street. He whistled.

$$
(x)(\operatorname{street}(x)) \text {. }
$$

As was mentioned before, indefinite noun phrases give rise to new discourse referents, and definite noun phrases are linked to existing discourse referents. The indefinite in the first sentence of (6) introduces a new discourse referent $y$ and two conditions $\operatorname{man}(y)$ and $y$ walked down the street. The second condition can be decomposed further by introducing a fresh discourse referent in the structure, linking this discourse referent to an existing discourse referent, and replacing the definite noun phrase with the discourse referent in two new conditions. This gives three new conditions all in all: $z=x, \operatorname{street}(z)$ and walked-down $(y, z)$. The discourse representation structure now looks like (8).

$$
(x, y, z)(\operatorname{street}(x), \operatorname{man}(y), z=x, \operatorname{street}(z), \text { walked-down }(y, z)) .
$$

Processing the second sentence of (1) gives rise to a new link and a new condition. The final result is (9).

$$
\begin{aligned}
& (x, y, z, u)(\operatorname{street}(x), \operatorname{man}(y), z=x, \operatorname{street}(z), \text { walked-down }(y, z), \\
& u=y, \text { whistled }(u)) .
\end{aligned}
$$

All representation conditions in the above example are atomic. Quantified noun phrases or logical operators such as conditionals or negations give rise to complex conditions. The representation structure for (4) given in (10) provides an example.

$$
\begin{aligned}
& ((x, y)(\operatorname{man}(x), \text { woman }(y), \operatorname{attractive}(y), \text { meet }(x, y))) \\
& \Rightarrow((),(\operatorname{smiles}-\operatorname{at}(x, y))) .
\end{aligned}
$$

Note the appearance of an arrow $\Rightarrow$ between components of the structure, glueing two non-atomic pieces of representation together. Note also that the righthand component starts with an empty list (), to express that on the righthand side no new discourse referents get introduced.

In the box format that many people are perhaps more familiar with, (10) looks like (11).

\begin{tabular}{|l|}
\hline$x y$ \\
\hline man $x$ \\
woman $y$ \\
attractive $y$ \\
meet $(x, y)$
\end{tabular}$\Rightarrow$ smiles-at $(x, y)$

Formal definitions and truth conditions for these representation structures will be given in the next section. 
Kamp (1981) and Kamp \& Reyle (1990) spell out the rules for processing sentences in the context of a representation structure in all the required formal detail. An important feature of the rules is that they impose formal constraints on availability of discourse referents for anaphoric linking. Roughly, the set of available discourse referents consists of the discourse referents of the current structure, plus the discourse referents of structures that can be reached from the current one by a series of steps in the directions left, (i.e. from the consequent of a pair $R \Rightarrow R^{\prime}$ to the antecedent, and up, i.e. from a structure to an encompassing structure. The constraints on discourse referent accessibility are used to explain the awkwardness of anaphoric links as in (12).

*If every man $_{1}$ meets an attractive woman $_{2}$, he $e_{1}$ smiles at her 2 .

Such data can be disputed, but space does not permit such indulgence here. Discourse referents for proper names are always available for anaphoric reference; to reflect this fact, such discourse referents are always included in the list of discourse referents of the top level structure.

To account for deictic uses of pronouns, use is made of anchored structures. An anchored structure is a pair consisting of a representation structure $R$ and a function $f$, where $f$ is an anchor for a subset of the discourse referents of $R$, i.e. $f$ assigns appropriate individuals in a model to these discourse referents. For example, structure (7) could be anchored by mapping discourse referent $x$ to an appropriate street. Deictic pronouns are handled by linking them to anchored discourse referents.

Essentially the same approach to natural language analysis as was proposed in Kamp (1981) is advocated in Heim (1982). Heim uses the metaphor of a filing cabinet: the established representation structure $R$ is a file, and additions to the discourse effect a new structure $R^{\prime}$, which is the result of changing the file in the light of the new information (see the article DYNAMIC SEMANTICS).

The main program of discourse representation theory (in its generic sense) is an attempt to regard semantic interpretation as a dynamic process mapping representations plus contexts to new representations plus contexts. As Partee (1984) remarks, this shift from static semantics to dynamic semantics cum pragmatics means an enrichment of the enterprise of formal semantics, and should therefore make it easier to establish contact with other schools of semantics and/or pragmatics. Partee's prediction was proved correct in subsequent years by the widespread use of discourse representation theory in computational linguistics and by the application of techniques of anaphora resolution from Artificial Intelligence in systems based on discourse representation theory.

Discourse representation theory has also provided new inspiration to traditional Montague grammarians, who tend to be less than satisfied with the contextual rules for analysing discourse on the grounds that the influence of context make it difficult to work out what contribution individual phrases make to the meaning of the whole. A suitable dynamic perspective on the process of interpretation has shown these compositionality qualms to be unfounded, and discourse representation theory has been instrumental in bringing this dynamic turn about. See the article DYNAMIC SEMANTICS for details. 
Heim (1990) contains a perceptive appraisal of various alternatives to the approach of discourse representation theory (in its generic sense) to the problem of unbound anaphora.

\section{Discourse Representation Structures (DRSs)}

Formally, a discourse representation structure $\mathrm{R}$ consists of two parts: a finite list of discourse referents, and a finite list of conditions. The discourse referents in the list are called the discourse referents of $R$. The conditions of a structure $R$ may contain discourse referents that are not included in the list of discourse referents of $R$. Conditions can be atoms, links, or complex conditions. An atom is a predicate name applied to a number of discourse referents, a link is an expression $t=r$, where $r$ is a discourse referent, and $t$ is either a proper name or a discourse referent. The clause for complex conditions uses recursion: a complex condition is a condition of the form $R \Rightarrow R^{\prime}$, where $R$ and $R^{\prime}$ are discourse representation structures.

Next one defines truth for discourse representation structures with respect to a model. Call $M=\langle D, I\rangle$ an appropriate model for discourse representation structure $R$ if $I$ maps the discourse referents of $R$ to members of $D$, the $n$-place predicate names in the atomic conditions of $R$ to $n$-place relations on $D$, the names occurring in the link conditions of $R$ to members of $D$, and (here is the recursive part of the definition) $M$ is also appropriate for the structures in the complex conditions of $R$.

Let $M=\langle D, I\rangle$ be an appropriate model for structure $R$. An assignment in $M=\langle D, I\rangle$ is a mapping of discourse referents to elements of $D$. Assignment $f$ verifies $R$ in $M$ if there is an extension $f^{\prime}$ of $f$ with the following properties:

1. $f^{\prime}$ is defined for all discourse referents of $R$ and for all discourse referents occurring in atomic or link conditions of $R$.

2. If $P\left(r_{1}, \ldots, r_{n}\right)$ is an atomic condition of $R$ then $\left\langle f^{\prime}\left(r_{1}\right), \ldots, f^{\prime}\left(r_{n}\right)\right\rangle \in I(P)$.

3. If $t=r$ is a link condition of $R$ and $t$ and $r$ are both discourse referents, then $f^{\prime}(t)=f^{\prime}(r)$; if $t$ is a proper name and $r$ a discourse referent, then $I(t)=f^{\prime}(r)$.

4. If $R_{1} \Rightarrow R_{2}$ is a complex condition of $R$, then every assignment for $R_{1}$ which verifies $R_{1}$ and agrees with $f^{\prime}$ on all discourse referents that are not discourse referents of $R_{1}$ also verifies $R_{2}$.

A structure $R$ is true in $M$ if the empty assignment verifies $R$ in $M$. These definitions can be modified to take anchors into account in the obvious way, by focussing on assignments extending a given anchor.

Clearly, the expressive power of this basic representation language is quite limited. In fact, there is an easy recipe for translating representation structures to formulae of first order predicate logic. Assuming that discourse referents 
coincide with predicate logical variables, the atomic and link conditions of a representation structure are atomic formulae of predicate logic. The translation function ${ }^{\circ}$ which maps representation structures to formulae of predicate logic is defined as $R^{\circ}=\bigwedge C_{i}^{\circ}$, where $\bigwedge$ indicates a finite conjunction and the $C_{i}^{\circ}$ are the translations of the conditions of $R$. The translation for conditions is in turn given by the following clauses.

- For atomic conditions: $C^{\circ}=C$.

- For complex conditions: $\left(R_{1} \Rightarrow R_{2}\right)^{\circ}=\forall x_{1} \cdots \forall x_{n}\left(R_{1}^{\circ} \rightarrow \exists y_{1} \cdots \exists y_{m} R_{2}^{\circ}\right)$, where $x_{1}, \ldots, x_{n}$ is the list of discourse referents of $R_{1}$ and $y_{1}, \ldots y_{m}$ the list of discourse referents of $R_{2}$.

It is easy to show that $R$ is true in $M$ under the definition given above if and only if $R^{\circ}$ is true in $M$ for some assignment, under Tarski's definition of truth for first order predicate logic.

A slight extension of the discourse representation language allows for the treatment of negation. Negated conditions take the form $\neg R$, where $R$ is a discourse representation structure. Negations of atomic conditions are treated as negations of discourse representation structures containing just one atomic condition. The discourse referents of a negated structure are not available for anaphoric linking outside that structure.

The definition of satisfaction has to take negated conditions into account. Here is the required extension of the definition. Assignment $f$ verifies $R$ in $M$ if there is an extension $f^{\prime}$ of $f$ with the following properties:

1. - 4. As above.

5. If $\neg R^{\prime}$ is a complex condition of $R$, then no assignment which agrees with $f^{\prime}$ on all discourse referents that are not discourse referents of $R^{\prime}$ verifies $R^{\prime}$.

Translation into predicate logic now has to take care of negation as well. The translation clause for negated conditions runs as follows.

- $(\neg R)^{\circ}=\neg \exists x_{1} \cdots \exists x_{n} R^{\circ}$

Here $x_{1}, \ldots, x_{n}$ is the list of discourse referents of $R$. It is easy to see that the given translation is meaning preserving. It is also not difficult to give a meaning preserving translation in the other direction. This shows that the discourse representation language extended with negation has precisely the same expressive power as first order predicate logic.

\section{Extensions: Tense and Plurals}

Partee (1984) gives a survey of proposals to extend discourse representation theory with discourse referents for times and events to exploit the parallels between nominal and temporal anaphora. In example (5) from section 1, where first reference is made to a seeing event in the past, and then to an event of crossing 
the street which takes place immediately after the seeing event, an anchoring mechanism can be used to link the seeing event to the appropriate time, and an anaphoric link between events can constrain the time of the crossing event in the appropriate way. Also, the dynamic effect of shifting the reference time can be incorporated by using a designated discourse referent for the reference time and specifying that this discourse referent be updated as a side effect of the processing of sentences denoting events.

Next, there are examples where a reference is picked up to an indefinite time in the past.

Mary arrived during the day. She let herself into the house.

In example (13), the arrival takes place at some indefinite time on a specific day (presumably anchored) in the past. The event of Mary's entering the house is then linked to the time of arrival. Again, all that is needed is the introduction of an event discourse referent for the arrival event, and an appropriate linking of this event discourse referent to the reference time discourse referent: the reference time discourse referent starts pointing at a time interval just after the time of arrival. The processing of the next sentence introduces an event that is constrained to be included in the reference time interval, and has again as a side effect that the reference time discourse referent gets shifted to refer to a time interval just after the house entering event.

Sentence (14) provides an example of quantification over times.

When Bill called, Mary was always out.

The example gives rise to a complex representation of the form $R \Rightarrow R^{\prime}$, with an event discourse referent and a reference time discourse referent introduced in the lefthand structure, and a state discourse referent of the righthand structure, with the state constrained to include the reference time interval.

An operator account of tenses and temporal adverbs has the awkwardness that the tense operator is redundant if a temporal adverb is present, as in (15), but not otherwise. Also, assigning the correct scopes to these operators poses problems.

$$
\text { Bill called last Friday around noon. }
$$

In the discourse representation approach, where tenses translate into event or state variables linked to an appropriate reference time, temporal operators are simply translated as predications on the event discourse referent, and the awkwardness vanishes. See Kamp \& Rohrer (1983) and Partee (1984), plus the references cited there, for details.

As for the incorporation of the singular/plural distinction, an obvious first move in any attempt to accommodate plural anaphoric pronouns is to make a distinction between singular and plural discourse referents. Singular pronouns are linked to singular discourse referents, plural pronouns to plural discourse referents. Plural indefinite noun phrases (some women, three men) introduce 
plural discourse referents, but it turns out that many other introduction mechanisms have to be postulated to get a reasonable coverage of plural anaphoric possibilities.

Plural discourse referents may result from summation of singular discourse referents. This is to account for uses of they that pick up a reference to a set of individuals which have been introduced one by one. Next, plural individuals may be the result of abstraction from complex conditions. Comsider example (16).

John bought every book Mary had mentioned.

He started reading them straight away.

Obviously, them refers to the set of all books mentioned by Mary. No plural discourse referent is introduced by the first sentence, so the only way to make one available is by calling it into being through abstraction.

So-called dependent plurals should be handled differently again, because here the plurality seems closely linked to syntax. Sentence (17) provides an example.

All my friends have children.

It is clear that (17) is still true if each of my friends has exactly one child. Dependent plurals call for a kind of in-between discourse referent which is neutral between singular and plural. The chapter on plurals in Kamp \& Reyle (1990) gives a very detailed account of these and related matters. The article PLURALITY provides further information on general issues of the interpretation of plurals.

\section{Incorporating Generalized Quantifiers}

Extending discourse representation theory with non standard quantifiers, and then getting the truth conditions right, is not completely straighforward.

Most farmers who own a donkey beat it.

Applying a routine strategy for building a representation structure for example (18), one arrives at structure (19), where $R \Rightarrow_{m} \Rightarrow R^{\prime}$ is true if most verifying assignments for $R$ are verifying assignments for $R^{\prime}$.

$$
((x, y)(\operatorname{farmer}(x), \operatorname{donkey}(y), \text { own }(x, y))) \Rightarrow_{m} \Rightarrow(()(\operatorname{beat}(x, y))) \text {. }
$$

This analysis does give the wrong truth conditions, because it quantifies over farmer-donkey pairs instead of individual farmers. In a situation where there are five kind farmers who each own one donkey and treat it well, and one cruel, rich farmer who beats each of his ten donkeys, the analysis makes sentence (18) true, while intuitively it should be false in this situation. 
The remedy (proposed in Kamp \& Reyle (1990)) involves a complication in the notation. Generalized quantifiers are introduced explicitly in the representation structures. The revised representation for (18) is (20).

$$
((x, y)(\text { farmer }(x), \operatorname{donkey}(y), \text { own }(x, y))) \Rightarrow_{x}^{\text {most }} \Rightarrow(()(\operatorname{beat}(x, y))) \text {. }
$$

At the place of most in (20) one could in principle have any generalized quantifier (see the article QUANTIFIERS, SEMANTICS OF). In other words, for every binary generalized quantifier $Q$ and every pair of representation structures $R, R^{\prime}$, the following is a complex condition: $R \Rightarrow_{v}^{Q} \Rightarrow R^{\prime}$. The truth conditions are modified to reflect what is expressed by the quantifier $Q$. Generalized quantifiers expres relations between sets, so $R \Rightarrow{ }_{x}^{Q} \Rightarrow R^{\prime}$ is true in case the two sets are in the appropriate quantifier relation. The truth conditions must pick out the two relevant sets. Here is the new part of the definition. Assignment $f$ verifies $R$ in $M$ if there is an extension $f^{\prime}$ of $f$ with the following properties:

1. - 5. As above.

6. If $R_{1} \Rightarrow \underset{v}{Q} \Rightarrow R_{2}$ is a complex condition of $R$, then $f^{\prime}$ verifies this condition if the sets $B$ and $C$ are in the quantifier relation denoted by $Q$, where $B=$ $\left\{b \mid f^{\prime}\right.$ has an extension $g$ with $g(v)=b$ which verifies $R_{1}$ in $\left.M\right\}$ and $C=$ $\left\{c \mid f^{\prime}\right.$ has an extension $h$ with $h(v)=c$ which verifies $R_{1}$ and $R_{2}$ in $\left.M\right\}$.

It is left to the reader to check that this gets the truth conditions for (18) right.

The following representation of (18) brings the incorporation of generalized quantifiers more in line with standard logical notation:

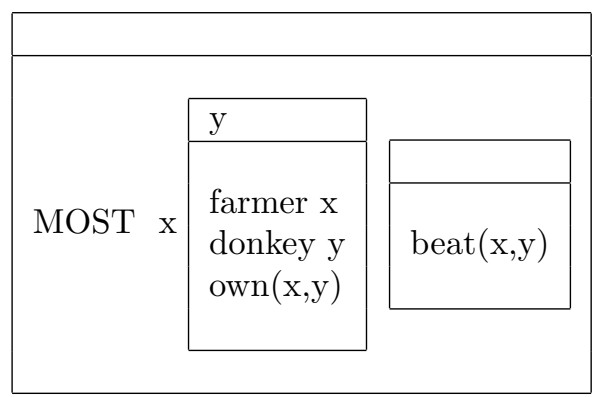

\section{Discourse Structures and Partial Models}

There is more than an occasional hint in the original papers of Kamp and Heim that discourse representation structures are closely connected to partial models. If the suggestion is not that these representation structures are themselves partial models, it is at least that they are intended to be interpreted with respect to partial models. That the structures are themselves partial models cannot be right: complex conditions are constraints on models rather than model components. They specify general properties that a model must have to satisfy the condition. Interpretation of discourse representation structures in partial 
models has never really been worked out. The truth definitions for representation structures, e.g. in Heim (1982), Kamp (1981), Kamp \& Reyle (1990) define satisfaction in classical (i.e. 'complete') models.

Because the representation structures contain identity links and negated identity links, evaluation in partial models where not only the predicates used to translate the vocabulary of the fragment, but also the identity predicate receives a partial interpretation, is feasible. Interestingly, this sheds light on some puzzling aspects of identity statements. Current studies of partial model theory interpret identity as a total predicate (see Langholm (1988)) Partializing identity leads to a more radical form of partially; it has as its effect that the objects in the model are not proper individuals but rather proto-individuals that can still fuse into the same individual after some more information acquisition about the identity relation. Technically, this form of radical partiality can be implemented by evaluating discourse representation structures with respect to models where the identity relation is a partial relation.

The formal development of a theory of partial identity involves an interpretation of identity as a pair $\left\langle I^{+}, I^{-}\right\rangle$, with $I^{+}$an equivalence relation which denotes the positive extension of identity, and $I^{-}$an anti-equivalence relation, that is to say a relation which is irreflexive, symmetric and anti-transitive, i.e. satisfying the requirement that if $I^{-} x y$ then it holds for all $z$ that $I^{-} x z$ or $I^{-} z y$.

The assumption that proto-individuals rather than regular individuals populate the partial models is attractive from the point of view of knowledge representation: often human beings have only partial information about identities. Famous paradoxes and puzzles are based on this fact. One example is Frege's morning star, evening star paradox; see the article COREFERENCE, IDENTITY AND SIMILARITY. Another is Saul Kripke's Pierre puzzle. Pierre is a Frenchman who has read about a famous and wonderful city he knows as Londres, and because of his reading he thinks that Londres is pretty. Later on he is abducted and forced to work in a slum in a city that, as he learns, is called London, and this new experience leads him to conclude that London is ugly. The important point to note is that as long as all this information is processed with respect to a partial model where London and Londres name different protoindividuals, Pierre's beliefs are not incoherent. They only become incoherent once it is discovered that London and Londres are identical, i.e. once Pierre acquires additional information about the extension of the identity relation. From outside, from a situation where London and Londres are anchored to the same individual, the belief may seem incoherent as well, but the point is that Pierre does not have full information about the nature of this anchor. The example is discussed in the context of discourse representation theory in Asher (1986), but the solution proposed there is still phrased in terms of classical models.

\section{Reasoning with DRSs}

The plausibility of using Discourse Representation Structures to model belief and other propositional attitudes is closely connected with the existence of cog- 
nitively plausible inference systems for DRSs. Proof theories for DRSs are given in Saurer (1993), Kamp and Reyle (1996), and Van Eijck (1999). The calculus of Van Eijck (1999) is perhaps the simplest of these, and we present it here.

We switch to the version of DRT where DRS negation is primitive and $D_{1} \Rightarrow D_{2}$ is defined in terms of negation. The precise definition is given below. A slight modification of the DRS definition is to make a distinction between the fixed discourse referents and the introduced discourse referents of a DRS (first proposed in Visser 1994). This allows for a natural definition of DRT consequence. If a DRS is inferred, its fixed discourse referents are supposed to be supplied by the premisses of the inference. They are 'fixed by the context of the inference', so to speak.

Thus, we view a DRS as a triple consisting of a set of fixed referents $F$, a set of introduced referents $I$, and a set of conditions $C_{1} \cdots C_{n}$ constrained by the requirement that the free variables in the $C_{i}$ must be among $F \cup I$. Concretely, the syntax of DRT looks like this (equality statements left out for simplicity of exposition):

\section{Definition 1 (DRT)}

$$
\begin{aligned}
t & ::=c \mid v \\
C & ::=\top\left|P t_{1} \cdots t_{n}\right| \neg D \\
D & ::=\frac{v_{1} \cdots v_{n}}{C_{1} \cdots C_{k+1} \cdots v_{m}}
\end{aligned}
$$

We will use $\perp$ as an abbreviation of $\neg T$. The (active) discourse referents of a term $t$ or condition $C$ or DRS $D$ are given by:

$$
\begin{aligned}
M(v):= & \{v\}, M(c):=\emptyset, M(\top):=\emptyset, M\left(P t_{1} \cdots t_{n}\right):=\cup_{i} M\left(t_{i}\right), \\
& M\left(\begin{array}{c|c|c|}
F & I \\
\hline C
\end{array}\right):=F \cup I, M\left(\neg \begin{array}{c|c|}
F & I \\
\hline & C
\end{array}\right):=F .
\end{aligned}
$$

Conditions on the formation rule for a DRS \begin{tabular}{c|c|c|}
\cline { 2 - 3 } & $v_{1} \cdots v_{n}$ & $v_{n+1} \cdots v_{m}$ \\
\hline$C_{1} \cdots C_{k}$ & :
\end{tabular}

1. $\left\{v_{1} \cdots v_{n}\right\} \cap\left\{v_{n+1} \cdots v_{m}\right\}=\emptyset$,

2. $\cup_{i} M\left(C_{i}\right) \subseteq\left\{v_{1} \cdots v_{m}\right\}$.

We define the condition

$$
\begin{array}{|l|c|}
\hline F & I \\
\hline C_{1} \cdots C_{n} \\
\hline
\end{array}
$$

as

$$
\neg \begin{array}{|l|c|}
\hline F & I \\
\hline C_{1} \cdots C_{n}, \neg D \\
\hline
\end{array}
$$

Here is a semantics for DRT in terms of partial assignments, following the original set-up in Kamp (1981). 
Figure 1: The Calculus for DRT

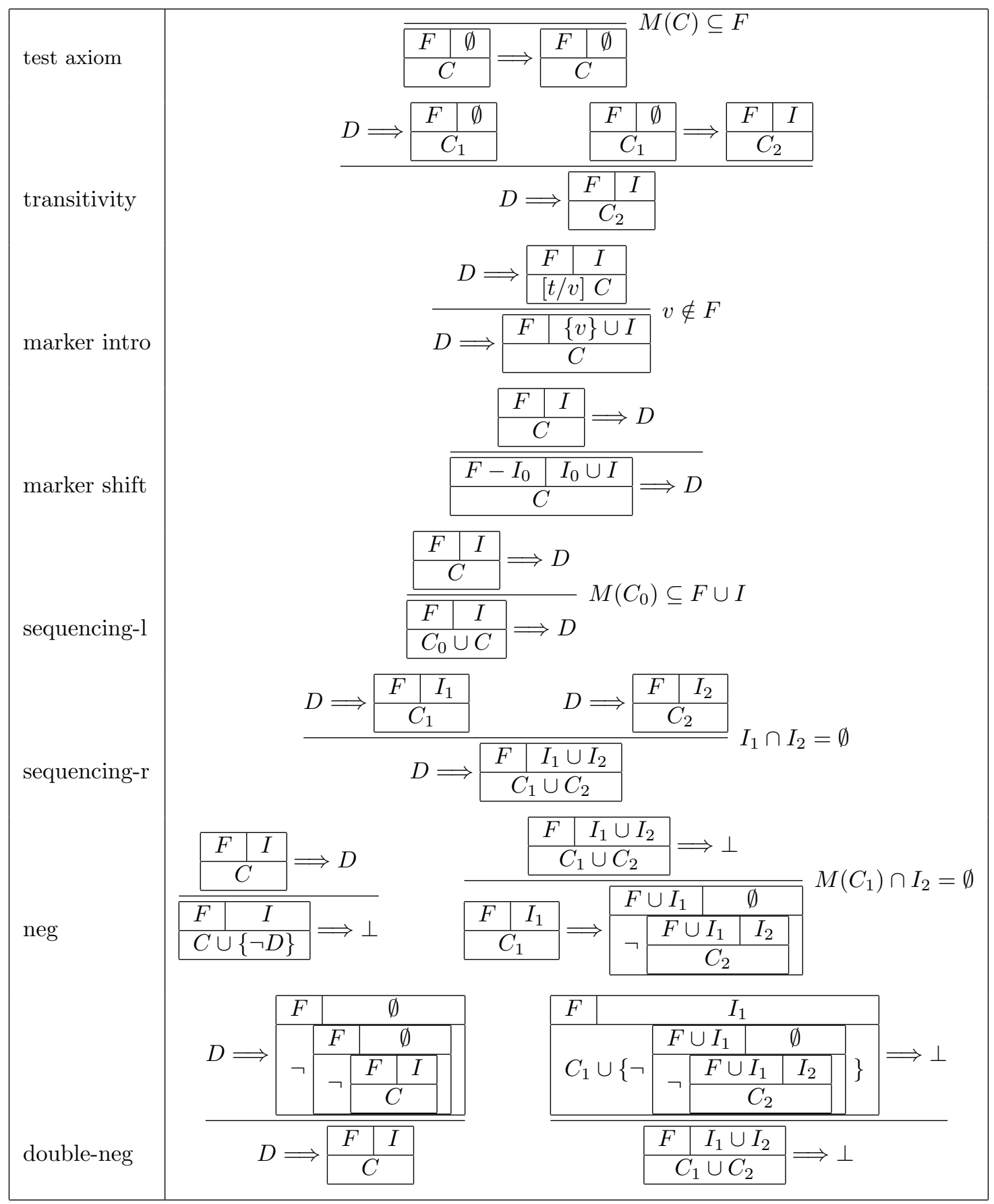




\section{Definition 2 ( Semantics of DRT)}

$$
\begin{aligned}
& \mathcal{M}, f=\top \\
& \mathcal{M}, f=P t_{1} \cdots t_{n} \\
& \mathcal{M}, f \models \neg D \\
& \text { iff }\left\langle\llbracket t_{1} \rrbracket_{f}^{\mathcal{M}}, \ldots, \llbracket t_{n} \rrbracket_{f}^{\mathcal{M}}\right\rangle \in P^{\mathcal{M}} \\
& \text { iff there is no } g \text { with } \mathcal{M}, f, g=D \\
& \mathcal{M}, f, g \models \begin{array}{|l|l|}
\hline F & I \\
\hline C_{1} \cdots C_{n} \\
\hline
\end{array} \\
& f: F \rightarrow \operatorname{dom}(\mathcal{M}) \text {, } \\
& \text { iff } \quad g: F \cup I \rightarrow \operatorname{dom}(\mathcal{M}), f=g \uparrow F \text {, } \\
& M, g \models C_{1}, \ldots, M, g \models C_{n} \text {. }
\end{aligned}
$$

Here $g \uparrow F$ denotes the restriction of function $g$ to the set $F$. The following definition of DRT consequence makes essential use of the distinction between fixed and introduced discourse referents.

\section{Definition 3 (DRT Consequence)}

$$
\begin{array}{|l|c|}
\hline F & I \\
\hline C_{1} \cdots C_{n} \\
\hline
\end{array} \mid \begin{array}{|l|c|}
\hline F \cup I & I^{\prime} \\
\hline C_{n+1} \cdots C_{m} \\
\hline
\end{array}
$$

iff for all $\mathcal{M}, f, g$ with $\mathcal{M}, f, g \models$\begin{tabular}{|l|c|}
\hline$F$ & $I$ \\
\hline$C_{1} \cdots C_{n}$ \\
\hline
\end{tabular}

there is an $h$ with $\mathcal{M}, g, h=$\begin{tabular}{|l|l|}
\hline$F \cup I$ & $I^{\prime}$ \\
\hline$C_{n+1} \cdots C_{m}$ \\
\hline
\end{tabular} .

A DRT calculus is given in Figure 1 (lists $C_{1} \cdots C_{k}$ abbreviated as $C$ ). The calculus uses substitution in constraints and DRSs. This notion is defined by:

$$
\begin{aligned}
& {[t / v] \top:=\top} \\
& {[t / v] P t_{1} \cdots t_{n} \quad:=P[t / v] t_{1} \cdots[t / v] t_{n},} \\
& {[t / v] \neg D:=\neg[t / v] D} \\
& {[t / v] \begin{array}{|l|c|}
\hline F & I \\
\cline { 2 - 3 } & C_{1} \cdots C_{n} \\
\cline { 2 - 2 }
\end{array}:=\begin{array}{|l|l|}
\hline F & I \\
\hline[t / v] & C_{1} \cdots[t / v] C_{n} \\
\hline
\end{array}}
\end{aligned}
$$

Of course, when a substitution $[t / v]$ is mentioned in a rule, it is assumed that $t$ is free for $v$ in $D$. It is also assumed that all DRSs mentioned in the rules satisfy the syntactic wellformedness conditions for DRSs.

Theorem 4 The calculus for DRT is sound.

Proof. Induction on the basis that the test axiom is sound and that the rules preserve soundness. Here is one example soundness check, for the rule of marker introduction. Assume $\mathcal{M}, f, g \models D$. Then by the soundness of the

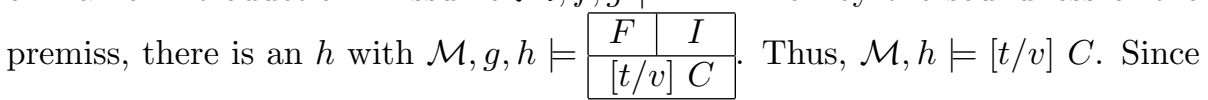
$v \notin F, h^{\prime}$ given by $h^{\prime}(v)=\llbracket t \rrbracket_{h}^{\mathcal{M}}$, and $h^{\prime}(w)=h(w)$ for all $w \neq v$ for which $h$ is defined extends $g$. By (an appropriate DRT version of) the substitution lemma, $\mathcal{M}, g, h^{\prime}=$\begin{tabular}{|c|c|c|}
\hline$F$ & $\{v\} \cup I$ \\
\hline & $C$
\end{tabular} . This proves $D \models$\begin{tabular}{|l|l|}
$F$ & $\{v\} \cup I$ \\
\hline$C$
\end{tabular}. 
Theorem 5 The calculus for DRT is complete.

For the proof of this we refer to Van Eijck (1999), where the proof system for DRT is related to a proof system for dynamic predicate logic (Groenendijk \& Stokhof 1991).

\section{The Treatment of Ambiguities}

If an expression of a formal language is viewed as a tree, a partial specification of how the expression is built up from its components can be given by means of a description of constraints on syntax tree construction.

This is the approach to the treatment of ambiguities taken in Underspecified DRT or UDRT (Reyle 1993). In UDRT, a DRS is viewed as a tree, and an UDRS as a set of constraints on tree formation.

$$
\text { All students found most solutions. }
$$

To represent the scope ambiguity between the two quantifiers in (22), one needs a representation that is 'in between' the following two DRSs:
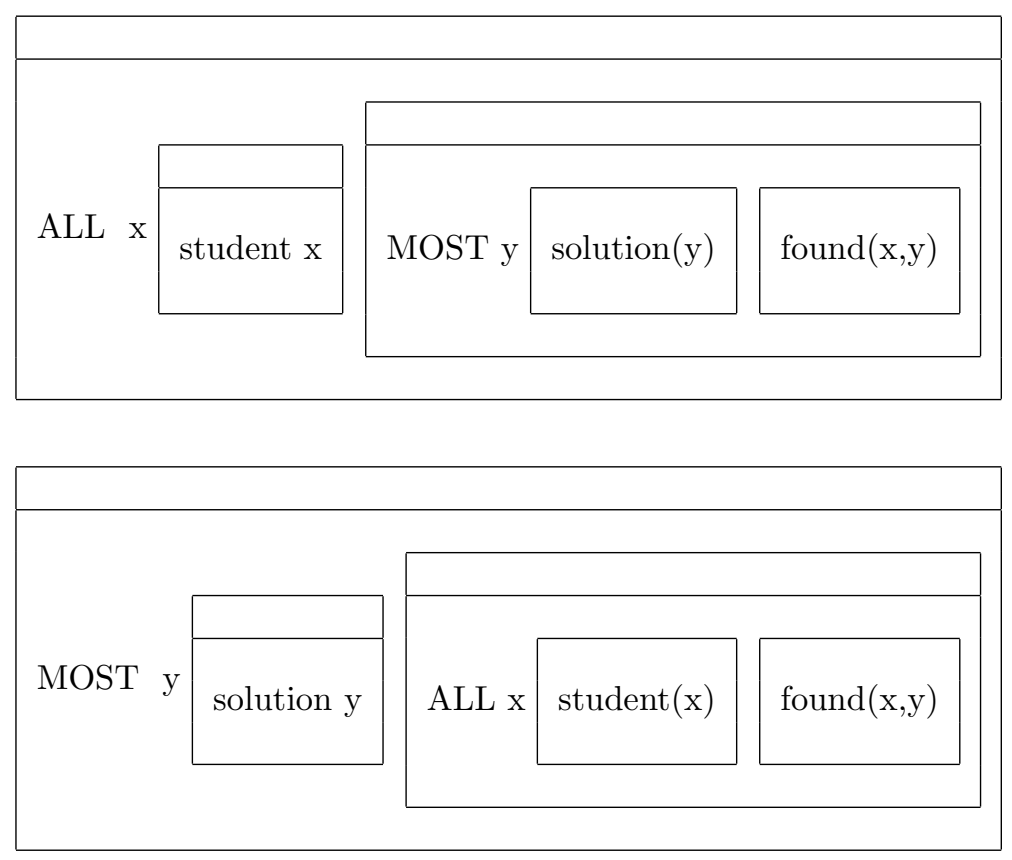

The UDRT solution is to take the DRSs apart, to label the parts, and to define an UDRS as a set of labelled DRS parts plus a list of constraints between labels. An UDRS for the example case has a top node $T$ labelled $l_{0}$, nodes

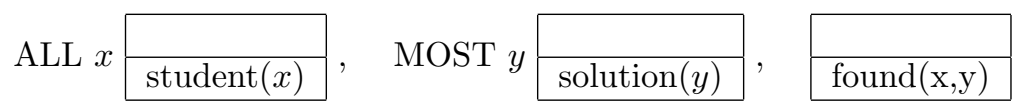


labelled $l_{1}, l_{2}, l_{3}$, respectively, and constraints $l_{0} \leq l_{1}, l_{0} \leq l_{2}, l_{1} \leq l_{3}, l_{2} \leq l_{3}$. Full disambiguation can be achieved by adding a further constraint. Adding the constraint $l_{1} \leq l_{2}$ disambiguates the UDRS to (23), while adding the constraint $l_{2} \leq l_{1}$ results in disambiguation to $(24)$.

\section{Keywords}

Dynamic semantics, discourse representation, discourse referent, context change, anaphora, anaphoric linking, quantification, Montague grammar, tense, plurality, belief ambiguity, representation of knowledge and belief.

\section{Cross References}

- ANAPHORA,

- ANAPHORA RESOLUTION,

- ANAPHORA, PHILOSOPHICAL ASPECTS OF -,

- UNDERSPECIFICATION,

- CONTEXT AND COMMON GROUND,

- DONKEY SENTENCES,

- FORMAL SEMANTIDS,

- PROPOSITIONAL ATTITUDES,

- MONTAGUE GRAMMAR,

- DYNAMIC SEMANTICS,

- QUANTIFIERS, SEMANTICS OF -

- PLURALITY,

- COREFERENCE, IDENTITY AND SIMILARITY

\section{BIO}

Jan van Eijck is Senior Researcher at CWI (Centre for Mathematics and Computer Science), Amsterdam, and professor of computational linguistics at UilOTS (Research Institute for Language and Speech), Utrecht. His main interests are applications of logic in natural language analysis and uses of logic in the analysis of communication and computation. 


\section{Bibliography}

Asher N 1986 'Belief in Discourse Representation Theory', Journal of Philosophical Logic, 15, 127-189.

Eijck J van 1999 'Axiomatising Dynamic Logics for Anaphora', Journal of Language and Computation, 1, 103-126.

Eijck J van \& H Kamp 1997 'Representing Discourse in Context', Handbook of Logic and Language, Elsevier, Amsterdam, 179-237

Geach P T 1962 Reference and Generality / an Examination of some Medieval and Modern Theories, Cornell University Press, Ithaca \& London (third revised edition: 1980).

Groenendijk J \& M Stokhof 1991 'Dynamic Predicate Logic', Linguistics and Philosophy, 14, 39-100.

Heim I R 1982 The Semantics of Definite and Indefinite Noun Phrases, Ph.D.. dissertation, University of Massachusetts, Amherst. Published 1987, Garland Press, New York.

Heim I R 1990 'E-Type Pronouns and Donkey Anaphora', Linguistics and Philosophy, 13, 137-177.

Kamp H 1981 'A Theory of Truth and Semantic Representation', in Groenendijk, Janssen, Stokhof (eds.), Formal Methods in the Study of Language, Mathematisch Centrum, Amsterdam.

Kamp H \& C Rohrer 1983 'Tense in Texts'. in Bäuerle, Schwarze \& Von Stechow (eds.), Meaning, Use and Interpretation of Language, Walter de Gruyter, Berlin, 250-269.

Kamp H \& U Reyle 1990 From Discourse to Logic / An Introduction to the Modeltheoretic Semantics of Natural Language, Formal Logic and Discourse Representation Theory, Kluwer, Dordrecht.

Kamp H \& U Reyle 1996 'A Calculus for First Order Discourse Representation Structures', Journal of Logic, Language and Information, 5, 297-348.

Kripke S 1979 'A Puzzle about Belief', in: A Margalit (ed.), Meaning and Use, Reidel, Dordrecht.

Langholm T 1988 Partiality, Truth and Persistence, CSLI Lecture Notes Number 15, Stanford University (distributed by University of Chicago Press).

Partee B H 1984 'Nominal and Temporal Anaphora', Linguistics and Philosophy, 7, 243-286.

Reyle U 1993 'Dealing with Ambiguities by Underspecification: Construction, Representation and Deduction', Journal of Semantics, 10, 123-179. 
Saurer W 1993 'A Natural Deduction System of Discourse Representation Theory', Journal of Philosophical Logic, 22, 249-302.

Seuren P A M 1985 Discourse Semantics, Basil Blackwell, Oxford.

Visser A 1994 Digressions on and desiderata for the design of dynamic discourse denotations, Lecture Notes, Department of Philosophy, Utrecht University. 